\title{
On stochastic reaction-diffusion equations with singular force term
}

\author{
AURELI ALABERT ${ }^{1}$ and ISTVÁN GYÖNGY ${ }^{2}$ \\ ${ }^{1}$ Departament de Matemàtiques, Universitat Autònoma de Barcelona, 08193 Bellaterra, Spain \\ ${ }^{2}$ University of Edinburgh, Department of Mathematics and Statistics, King's Buildings, \\ EdinburghEH9 3JZ, UK. E-mail: gyongy@maths.ed.ac.uk
}

We prove an existence and uniqueness theorem for stochastic reaction-diffusion equations driven by space-time white noise in one spatial dimension, when the diffusion coefficient is non-degenerate and the force term is only measurable and can be locally unbounded.

Keywords: space-time white noise; stochastic reaction-diffusion equations

\section{Introduction}

Differential equations may be ill behaved in the sense that they have no solutions, or nonunique solutions, or solutions which do not depend continuously on the given data. Surprisingly, by introducing some random perturbation we can often transform these equations into well-behaved stochastic differential equations. This phenomenon is well known for ordinary differential equations. In the present paper we are interested in this regularization effect of the noise on ill-posed partial differential equations (PDEs). In order to illustrate the result of the paper, let us consider the following examples of simple reaction-diffusion equations:

$$
\begin{aligned}
\frac{\partial u}{\partial t}(t, x) & =\frac{\partial^{2} u}{\partial x^{2}}(t, x)+2 \sqrt{\sin (\pi x) u(t, x)}+\pi^{2} u(t, x), \quad t \geqslant 0, \quad x \in(0,1), \\
u(t, 0) & =u(t, 1)=0, \quad t \geqslant 0, \\
u(0, x) & =0, \quad x \in[0,1] \\
\frac{\partial u}{\partial t}(t, x) & =\frac{\partial^{2} u}{\partial x^{2}}(t, x)-f(u(t, x)), \quad t \geqslant 0, \quad x \in(0,1) \\
u(t, 0) & =u(t, 1)=0, \quad t \geqslant 0 \\
u(0, x) & =1, \quad x \in[0,1],
\end{aligned}
$$

where $f:=z^{-1 / 3}$ for $z \neq 0$ and $f(0)=1$.

Clearly, both $u: \equiv 0$ and $v:=t^{2} \sin (\pi x)$ solve the first example. Hence one can easily construct infinitely many solutions. On the other hand, by adding a 'multiplicative noise' 
$\sigma(u(t, x)) \partial^{2} W / \partial t \partial x$ to the right-hand side of (1.1), we transform it into a stochastic PDE which has a unique solution for any small non-degenerate Lipschitz function $\sigma$ and for any given space-time white noise $\partial^{2} W / \partial t \partial x$ on $[0, \infty) \times[0,1]$. This result is known from Bally et al. (1994), where an existence and uniqueness theorem is proved for stochastic reaction-diffusion equations which locally bounded measurable force terms. The proof of this result in Bally et al. (1994) is based on a priori $L^{p}$-estimates, obtained by Malliavin calculus for the density of the solution. In Gyöngy (1998) it is shown that existence and uniqueness results hold also for a larger class of non-degenerate stochastic semilinear PDEs with locally bounded force terms, even if no density estimates, like those from Bally et al. (1994), are available.

The second example does not have any solution, because the local solution is 'trapped' by the singularity of $f$. Note that $f$ is locally unbounded, so the previous results do not tell us if random perturbations with non-degenerate multiplicative noises can compensate its irregularity. As an application of the main result (Theorem 2.1) of the present paper, we obtain that these perturbations also regularize (1.2). A similar result is proved in Gyöngy and Pardoux (1993) for the special case of random perturbations with additive space-time white noise. In the proof of Theorem 2.1 we use ideas and results from Bally et al. (1994) and Gyöngy and Pardoux (1993). The main difficulty is to obtain the necessary a priori $L^{p_{-}}$ estimates for the Green measure of the solution. To prove the uniqueness of the solution is more delicate here than it is in Gyöngy and Pardoux (1993) and Bally et al. (1994), and we can obtain the uniqueness only in the class of solutions satisfying an integrability condition ((2.5) below).

For standard definitions and tools of the theory of stochastic PDEs we refer to Da Prato and Zabczyk (1992), Rozovskii (1990) and Walsh (1986).

\section{Formulation of the problem and the main results}

Let $\left(\Omega, \mathscr{F},\left\{\mathscr{F}_{t}\right\}_{0 \leqslant t \leqslant T}, P\right)$ be a filtered probability space carrying an $\mathscr{F}_{t}$-Brownian sheet $W=W(t, x)$ on $[0, T] \times[0,1]$. This means $W$ is a Gaussian field, $\mathrm{E} W(t, x)=0$, $\mathrm{E}(W(t, x) W(s, y))=(t \wedge s)(x \wedge y), \quad W(t, x)$ is $\mathscr{F}_{t}$-measurable and $W(t, x)-W(s, x)+$ $W(s, y)-W(t, y)$ is independent of $\mathscr{F}_{s}$ for all $0 \leqslant s \leqslant t$ and $x, y \in[0,1]$.

Let $f=f(t, x, z)$ and $\sigma=\sigma(t, x, z)$ be $\mathscr{F}_{t}$-adapted random fields on $[0, \infty) \times$ $[0,1] \times \mathbb{R}$. This means that they are functions of $(t, \omega, x, z) \in[0, \infty) \times \Omega \times[0,1] \times \mathbb{R}$, such that they are Borel-measurable in $(t, x, z)$ and $\mathscr{F}_{t}$-adapted for fixed $x, z$. Let $u_{0}=u_{0}(x)$ be a continuous $\mathscr{F}_{0}$-measurable random field such that $u_{0}(0)=u_{0}(1)=0$. We consider the Dirichlet problem

$$
\begin{aligned}
\frac{\partial u}{\partial t}(t, x) & =\frac{\partial^{2} u}{\partial x^{2}}(t, x)+f(t, x, u(t, x))+\sigma(t, x, u(t, x)) \frac{\partial^{2} W}{\partial t \partial x}, \quad t \geqslant 0, \quad x \in(0,1), \\
u(t, 0) & =u(t, 1)=0, \quad t \geqslant 0, \\
u(0, x) & =u_{0}, \quad x \in[0,1],
\end{aligned}
$$


denoted by $\operatorname{Eq}\left(u_{0}, f, \sigma\right)$. We say that a random field $u=u(t, x)$ is a solution to $\operatorname{Eq}\left(u_{0}, f, \sigma\right)$ on $[0, T]$, if $u(t, x)$ is $\mathscr{F}_{t}$-measurable for every $t, x$, it is continuous in $(t, x) \in$ $[0, T] \times[0,1]$, and almost surely (a.s.)

$$
\begin{aligned}
\int_{0}^{1} u(t, x) \varphi(x) \mathrm{d} x= & \int_{0}^{1} u_{0}(x) \varphi(x) \mathrm{d} x+\int_{0}^{t} \int_{0}^{1} u(s, x) \varphi^{\prime \prime}(x) \mathrm{d} x \mathrm{~d} s+\int_{0}^{t} \int_{0}^{1} f(s, x, u(s, x)) \varphi(x) \mathrm{d} x \mathrm{~d} s \\
& +\int_{0}^{t} \int_{0}^{1} \sigma(s, x, u(s, x)) \varphi(x) \mathrm{d} W(s, x)
\end{aligned}
$$

for all $t \in[0, T]$ and $\varphi \in C^{2}([0,1]), \varphi(0)=\varphi(1)=0$, where the last integral on the rightside of this equality is understood as Itô's integral.

It is well known that $\operatorname{Eq}\left(u_{0}, f, \sigma\right)$ has a unique solution when $f$ and $\sigma$ are Lipschitz functions satisfying a linear growth condition and $u_{0}$ is continuous (see, for example, Walsh 1986). In the present paper we study the case when $f$ is only measurable and may be locally unbounded. In order to formulate our main results we use the notation $L^{p q r}$ for the space of functions $h:[0, T] \times[0,1] \times \mathbb{R} \rightarrow \mathbb{R}$ with the norm

$$
\|h\|_{p q r}:=\left(\int_{0}^{T}\left(\int_{0}^{1}\left(\int_{\mathbb{R}}|h(t, x, z)|^{p} \mathrm{~d} z\right)^{q / p} \mathrm{~d} x\right)^{r / q} \mathrm{~d} t\right)^{1 / r}<\infty .
$$

We assume the following conditions on the data $f, \sigma$ and $u_{0}$ :

(A) $f=f(t, x, z)$ is a Borel function such that

$$
|f(t, x, z)|^{2} \leqslant C+F(t, x, z), \quad \mathrm{d} t \otimes \mathrm{d} x \otimes \mathrm{d} z \text { almost everywhere (a.e.) }
$$

with some constant $C \geqslant 0$ and a non-negative function $F \in L^{p q r}$ for some $p>1$, $q>2, r>4 q /(3 q-2)$.

(B) $\sigma:[0, T] \times[0,1] \times \mathbb{R} \rightarrow \mathbb{R}$ and $1 / \sigma, \partial \sigma / \partial z, \partial^{2} \sigma / \partial z^{2}$ are bounded Borel functions for every $T>0$.

(C) $u_{0}$ is an $\mathscr{F}_{0}$-measurable continuous random field on $[0,1], u_{0}(0)=u_{0}(1)=0$.

Theorem 2.1. Assume conditions $(A),(B)$ and $(C)$. Then $\mathrm{Eq}\left(u_{0}, f, \sigma\right)$ has a unique solution u satisfying

$$
\int_{0}^{T} F(t, x, u(t, x)) \mathrm{d} x \mathrm{~d} t<\infty \text { (a.s.) }
$$

for every T. This solution is a $C([0,1])$-valued Markov process.

Remark 2.2. Assume (A), (B) and (C). Assume that $f$ satisfies condition (A) not only with constant $C \geqslant 0$ and non-negative function $F \in L^{p q r}$, but also with some other constant $D \geqslant 0$ and non-negative function $G \in L^{\alpha \beta \gamma}$ in place of $C$ and $F$. Let $u$ and $v$ denote the solutions of $\operatorname{Eq}\left(u_{0}, f, \sigma\right)$ in the class of solutions satisfying (2.5) with $F$ and with $G$ (in place of $F$ ), respectively. Note that $|f|^{2} \leqslant 3(C \vee D)+F \wedge G$, and $H:=F \wedge G \in L^{p q r} \cap L^{\alpha \beta \gamma}$. 
Hence $u=v$ by Theorem 2.1, that is to say, our statement on the uniqueness of the solution does not depend on the particular function $F$ which satisfies (A) with the given function $f$.

Theorem 2.3. Assume that the data $\left(u_{0}, f, \sigma\right)$ satisfy conditions $(A),(B)$ and $(C)$. Let $\bar{f}$ be a Borel function satisfying condition $(A)$. Let $\bar{u}_{0}$ be an $\mathscr{F}_{0}$-measurable continuous random field on $[0,1], \bar{u}_{0}(0)=\bar{u}_{0}(1)=0$, such that $\bar{u}_{0}(x) \leqslant u_{0}(x)($ a.s. $)$ for every $x \in(0,1)$. Assume that

$$
\bar{f}(t, x, z) \leqslant f(t, x, z) \quad \mathrm{d} t \otimes \mathrm{d} x \otimes \mathrm{d} z \text { (a.e.) }
$$

Then almost surely $\bar{u}(t, x) \leqslant u(t, x)$ for all $t, x$ for the solutions $u$ and $\bar{u}$ of $\mathrm{Eq}\left(u_{0}, f, \sigma\right)$ and $\operatorname{Eq}\left(\bar{u}_{0}, \bar{f}, \sigma\right)$.

\section{Preliminaries}

Let $g=g(t, x)$ be an $\mathscr{F}_{t}$-adapted random field on $[0, T] \times[0,1]$, such that $g \in L^{2}([0, T] \times[0,1])$ (a.s.). Define

$$
Z:=\exp \left(\int_{0}^{T} \int_{0}^{1} g(t, x) W(\mathrm{~d} t, \mathrm{~d} x)-\frac{1}{2} \int_{0}^{T} \int_{0}^{1} g(t, x)^{2} \mathrm{~d} x \mathrm{~d} t\right), \quad \mathrm{d} \tilde{P}:=Z \mathrm{~d} P,
$$

and assume $\mathrm{E}(Z)=1$. Then, by Girsanov's theorem,

$$
\tilde{W}(t, x):=W(t, x)-\int_{0}^{t} \int_{0}^{x} g(s, y) \mathrm{d} y \mathrm{~d} s
$$

is an $\mathscr{F}_{t}$-Brownian sheet under the probability $\tilde{P}$ (see, for example, Gyöngy and Pardoux 1992).

We will use Girsanov's theorem to derive the existence of continuous modifications of random fields $u$ satisfying (2.2). In particular, we will make use of the following proposition.

Proposition 3.1. Assume $(A),(B)$ and $(C)$. Let $u=\{u(t, x), t \in[0, T] \times[0,1]\}$ be an $\mathscr{F}_{t^{-}}$ adapted bounded random field satisfying (2.2) almost surely for each $t \in[0, T]$, $\varphi \in C^{2}([0,1])$, with $\varphi(0)=\varphi(1)=0$. Assume that $\sigma=\sigma(t, x, z)$ is bounded, that it is Lipschitz in $z$ and that $\eta=\eta(t, x):=(f / \sigma)(t, x, u(t, x)) \in L^{2}([0, T] \times[0,1])($ a.s. $)$. Define

$$
Z:=\exp \left(\int_{0}^{T} \int_{0}^{1} \frac{f}{\sigma}(t, x, u(t, x)) \mathrm{d} W(t, x)-\frac{1}{2} \int_{0}^{T} \int_{0}^{1}\left|\frac{f}{\sigma}(t, x, u(t, x))\right|^{2} \mathrm{~d} x \mathrm{~d} t\right) .
$$

Then $u$ has a continuous modification which is a solution of $\mathrm{Eq}\left(u_{0}, f, \sigma\right)$ on $[0, T]$. 
Proof. Assume first that, for some constant $R$,

$$
\int_{0}^{T} \int_{0}^{1}\left|\frac{f}{\sigma}(t, x, u(t, x))\right|^{2} \mathrm{~d} x \mathrm{~d} t \leqslant R
$$

By Girsanov's theorem,

$$
\tilde{W}(t, x)=W(t, x)+\int_{0}^{t} \int_{0}^{x} \frac{f}{\sigma}(s, y, u(s, y)) \mathrm{d} y \mathrm{~d} s
$$

is an $\mathscr{F}_{t}$-Brownian sheet under $\tilde{P}$, and $u$ satisfies

$$
\int_{0}^{1} u(t, x) \varphi(x) \mathrm{d} x=\int_{0}^{1} u_{0}(x) \varphi(x) \mathrm{d} x+\int_{0}^{t} \int_{0}^{1} u(s, x) \varphi^{\prime \prime}(x) \mathrm{d} x+\int_{0}^{t} \int_{0}^{1} \sigma(s, x, u(s, x)) \varphi(x) \mathrm{d} \tilde{W}(s, x),
$$

$\mathrm{d} t \otimes \tilde{P}$ a.e. on $[0, T]$. It is known (see Walsh 1986), that there exists a modification $\tilde{u}$ of $u$ which is continuous and satisfies (3.12) for all $t$. Hence, (2.2) holds for all $t \in[0, T]$ with $\tilde{u}$ in place of $u, \tilde{P}$ and $P$ almost surely. Consequently, $\tilde{u}$ is a solution to $\operatorname{Eq}\left(u_{0}, f, \sigma\right)$ under $P$. The general case can be obtained by a standard stopping-time argument.

We will use the following results on existence, uniqueness and comparison of the solutions. For their proofs we refer to Bally et al. (1994) and Gyöngy (1998).

Proposition 3.2. Let $f=f(t, x, z)$ and $\sigma=\sigma(t, x, z)$ be bounded measurable functions. Assume that $\sigma$ satisfies condition $(B)$. Let $\xi=\xi(t, x)$ be an $\mathscr{F}_{t}$-adapted random field such that $\int_{0}^{T} \int_{0}^{1} \xi^{2}(t, x) \mathrm{d} x \mathrm{~d} t<\infty$ (a.s.). Then $\operatorname{Eq}\left(u_{0}, f+\xi, \sigma\right)$ has a unique solution for every $\mathscr{F}_{0}$-adapted continuous random field $u_{0}$ on $[0,1]$.

Proposition 3.3. Let equations $\operatorname{Eq}\left(u_{0}, f+\xi, \sigma\right)$ and $\operatorname{Eq}\left(\bar{u}_{0}, \bar{f}+\bar{\xi}, \sigma\right)$ satisfy the conditions of Proposition 3.2. Assume that

$$
\begin{aligned}
\bar{f}(t, x, z) & \leqslant f(t, x, z) \quad \mathrm{d} t \otimes \mathrm{d} x \otimes \mathrm{d} z \text { a.e., } \\
\bar{\xi}(t, x) & \leqslant \xi(t, x) \quad P \otimes \mathrm{d} t \otimes \mathrm{d} x \text { a.e. } \\
\bar{u}_{0} & \leqslant u_{0} \quad P \otimes \mathrm{d} x \text { a.e. }
\end{aligned}
$$

Then almost surely $\bar{u}(t, x) \leqslant u(t, x)$ for all $(t, x)$, where $u$ and $\bar{u}$ denote the solutions of $\mathrm{Eq}\left(u_{0}, f+\xi, \sigma\right)$ and $\operatorname{Eq}\left(\bar{u}_{0}, \bar{f}+\bar{\xi}, \sigma\right)$, respectively.

\section{Estimates and passage to the limit}

In this section we prove the estimates which we need in order to be able to pass to the limit in the equations which approximate $\operatorname{Eq}\left(u_{0}, f, \sigma\right)$. These estimates are stated in Proposition 4.3, which extends the corresponding estimates from Gyöngy and Pardoux (1993) to the case of equations with multiplicative noise. First, we establish two preliminary estimates (Lemmas 
4.1 and 4.2) for the solution of $\operatorname{Eq}\left(u_{0}, 0, \sigma\right)$. The proof in Gyöngy and Pardoux (1993) of the corresponding estimates when $\sigma=1$ is based on a decomposition of the solution $u$ into a random field $\xi$, which is Gaussian, and into another field $\eta$, which is independent of $\xi$. Such a decomposition is obviously not available in our more general case. We use the notation

$$
\|h(t)\|_{p q}:=\left(\int_{0}^{1}\left(\int_{\mathbb{R}}|h(t, x, z)|^{p} \mathrm{~d} z\right)^{q / p} \mathrm{~d} x\right)^{1 / q}
$$

for functions $h=h(t, x, z)$.

Lemma 4.1. Assume $(B)$ and $(C)$. Let $u$ be the solution to $\mathrm{Eq}\left(u_{0}, 0, \sigma\right)$. Then, for any $p>1$, $q>2, r>4 q /(3 q-2)$, there exists a constant $K$, depending only on $T, p, q, r$ and the supremum norms of $\sigma, 1 / \sigma, \partial \sigma / \partial z$ and $\partial^{2} \sigma / \partial z$, such that, for every Borel function $h \in L^{p q r}$ and every $s \in[0, T]$,

$$
\mathrm{E}\left(\int_{s}^{T} \int_{0}^{1} h(t, x, u(t, x)) \mathrm{d} x \mathrm{~d} t \mid \mathscr{F}_{s}\right) \leqslant K\left(\int_{s}^{T}\|h(t)\|_{p q}^{r} \mathrm{~d} t\right)^{1 / r}
$$

Proof. For every deterministic initial value $u_{0} \in C([0,1])$, it is known from Bally et al. (1994) that, for every $t>0, x \in(0,1)$, the random variable $u(t, x)$ has a density $p(t, x ; z)$ with respect to the Lebesgue measure $\mathbb{R}$. Moreover, by Lemmas 3.2, 3.4 and 3.5 in Bally et al. (1994),

$$
\int_{\mathbb{R}}|p(t, x ; z)|^{\rho} \mathrm{d} z \leqslant C\left\{1+t^{-[(1+\alpha) / 4+\varepsilon] \rho}(x \wedge(1-x))^{-[(2-\alpha) / 2+\varepsilon] \rho}\right\},
$$

for every $\rho \geqslant 1, \alpha \in(0,1)$ and $\varepsilon>0$, with a constant $C$. Hence, with some constant $L$,

$$
\begin{aligned}
K & :=\left(\int_{0}^{T}\left(\int_{0}^{1}\left(\int_{\mathbb{R}}|p(t, x ; z)|^{\rho} \mathrm{d} z\right)^{\kappa / \rho} \mathrm{d} x\right)^{\delta / \kappa} \mathrm{d} t\right)^{1 / \delta} \\
& \leqslant L+L\left(\int_{0}^{T} t^{-[(1+\alpha) / 4+\varepsilon] \delta} \mathrm{d} t\right)^{1 / \delta}\left(\int_{0}^{1}(x \wedge(1-x))^{-\kappa[(2-\alpha) / 2+\varepsilon]} \mathrm{d} x\right)^{1 / \kappa},
\end{aligned}
$$

which is finite if $\kappa<2, \delta<4 \kappa /(3 \kappa-2)$ for sufficiently small $\varepsilon>0$ and $\alpha$ sufficiently close to 1 . Thus by Hölder's inequality,

$$
\mathrm{E}\left(\int_{s}^{T} \int_{0}^{1} h(t, x, u(t, x)) \mathrm{d} x \mathrm{~d} t\right) \leqslant K\left(\int_{s}^{T}\|h(t)\|_{p q}^{r} \mathrm{~d} t\right)^{1 / r}
$$

for every Borel function $h \in L^{p q r}$, where $p, q$ and $r$ are the conjugate numbers to $\rho, \kappa$ and $\delta$, respectively. Consequently, we have (4.5) for every $p>1, q>2$ and $r>4 q /(3 q-2)$. For every deterministic initial condition $u_{0}$, the constant $K$ above depends only on $T, p, q, r$ and the supremum norms of $\sigma, 1 / \sigma, \partial \sigma / \partial z, \partial^{2} \sigma / \partial z^{2}$. Next, we show that in estimates of the 
form (4.5), the constant $K$ is independent of the deterministic initial value $u_{0}$. To this end, let $\bar{u}_{0}(t, x)$ be the solution of $\mathrm{Eq}\left(u_{0}, 0,0\right)$ and note that $\bar{u}(t, x):=u(t, x)-\bar{u}_{0}(t, x)$ is the solution of $\mathrm{Eq}(0,0, \bar{\sigma})$ with $\bar{\sigma}(t, x, z):=\sigma\left(t, x, z+\bar{u}_{0}(t, x)\right)$. Hence, setting $\bar{h}(t, x, z):=$ $h\left(t, x, z+\bar{u}_{0}(t, x)\right)$, from (4.5) we have

$$
\begin{aligned}
\mathrm{E}\left(\int_{s}^{T} \int_{0}^{1} h(t, x, u(t, x)) \mathrm{d} x \mathrm{~d} t\right) & =E\left(\int_{s}^{T} \int_{0}^{1} h\left(t, x, \bar{u}(t, x)+\bar{u}_{0}(t, x)\right) \mathrm{d} x \mathrm{~d} t\right) \\
& \leqslant K\left(\int_{s}^{T}\|\bar{h}(t)\|_{p q}^{r} \mathrm{~d} t\right)^{1 / r}=K\left(\int_{s}^{T}\|h(t)\|_{p q}^{r} \mathrm{~d} t\right)^{1 / r}
\end{aligned}
$$

by the shift invariance of the Lebesgue measure. We now show (4.2). To this end we take a sequence of $\mathscr{F}_{s}$-measurable continuous random fields $v_{n}=\left\{v_{n}(x): x \in[0,1]\right\}$ such that $v_{n} \rightarrow u(s, \cdot)$ a.s. in $C([0,1])$ and $v_{n} \in \Gamma_{n}$ for all $\omega \in \Omega$ for a finite subset $\Gamma_{n}$ of some dense set $\left\{w^{i}: i=1,2,3 \ldots\right\}$ in $C([0,1])$. Let $u_{n}=\left\{u_{n}(t, x): s \leqslant t, x \in[0,1]\right\}$ denote the solution of (2.1) with initial condition $v_{n}$ at $t:=s$. Then, for almost all $\omega \in \Omega$, we have $u_{n}(t, x) \rightarrow u(t, x)$ for all $t \geqslant s, x \in[0,1]$. Let $u_{n}^{i}=\left\{u_{n}^{i}(t, x): s \leqslant t, x \in[0,1]\right\}$ denote the solution of (2.1) with initial condition $w^{i}$ at $t:=s$. Then, by well-known properties of the conditional expectation and by estimate (4.5),

$$
\begin{aligned}
\mathrm{E}\left(\int_{s}^{T} \int_{0}^{1} h\left(t, x, u_{n}(t, x)\right) \mathrm{d} x \mathrm{~d} t \mid \mathscr{F}_{s}\right) & =\mathrm{E}\left(\int_{s}^{T} \int_{0}^{1} \sum_{\left\{i: w^{i} \in \Gamma_{n}\right\}} \mathbf{1}_{\left\{v_{n}=w^{i}\right\}} h\left(t, x, u_{n}^{i}(t, x)\right) \mathrm{d} x \mathrm{~d} t \mid \mathscr{F}_{s}\right) \\
& =\sum_{\left\{i: w^{i} \in \Gamma_{n}\right\}} \mathbf{1}_{\left\{v_{n}=w^{i}\right\}} \mathrm{E}\left(\int_{s}^{T} \int_{0}^{1} h\left(t, x, u_{n}^{i}(t, x)\right) \mathrm{d} x \mathrm{~d} t\right) \\
& \leqslant K \sum_{\left\{i: w^{i} \in \Gamma_{n}\right\}}\left(\mathbf{1}_{\left\{v_{n}=w^{i}\right\}}\|h\|_{p q r}\right)=K\|h\|_{p q r} .
\end{aligned}
$$

Letting $n \rightarrow \infty$, we obtain (4.2) for every continuous $h \in L^{p q r}$. For fixed $R>0$, let $\mathscr{H}_{R}$ denote the set of Borel functions $h=h(t, x, r)$ such that (4.2) holds with $h_{R}:=$ $k_{R}(r) h(t, x, r)$ in place of $h$, where $k_{R}(t, x, r):=\max (-|r / R|+1,0)$. Then, as we have proved above, $\mathscr{H}_{R}$ contains every bounded continuous function. Moreover, if $h$ is a pointwise limit of an increasing uniformly bounded sequence from $\mathscr{H}_{R}$, then $h$ also belongs to $\mathscr{H}_{R}$. Hence, by the monotone class theorem, $\mathscr{H}_{R}$ contains every bounded Borel function $h$ (see, for example, Dellacherie and Meyer 1978, p. 15). Let $h \in L^{p q r}$ be a non-negative Borel function. Then $h_{R L}:=\min \left(h_{R}, L\right)$ belongs to $\mathscr{H}_{R}$ for all constants $R, L>0$. Hence, letting first $L \rightarrow \infty$ and then $R \rightarrow \infty$ in (4.2) with $h_{R L}$ in place of $h$, we obtain (4.2) for $h$ by Fatou's lemma. Consequently, (4.2) holds for every Borel function $h \in L^{p q r}$.

The following lemma generalizes Proposition 3.2.5 from Gyöngy and Pardoux (1993).

Lemma 4.2. Assume $(B)$ and $(C)$. Let $u$ be a solution of $\mathrm{Eq}\left(u_{0}, 0, \sigma\right)$. Then, for any $\alpha>1$, 
$\beta>2, \gamma>4 \beta /(3 \beta-2)$, there exists a real analytic function $A: \mathbb{R}_{+} \rightarrow(0, \infty)$ depending only on $T, \alpha, \beta, \gamma$ and the supremum norms of $\sigma, 1 / \sigma, \partial \sigma / \partial z$ and $\partial^{2} \sigma / \partial z$, such that for every Borel function $h:[0, T] \times[0,1] \times \mathbb{R} \rightarrow \mathbb{R}, h \in L^{\alpha \beta \gamma}$,

$$
\mathrm{E}\left\{\exp \left(\int_{0}^{T} \int_{0}^{1} h(t, x, u(t, x)) \mathrm{d} x \mathrm{~d} t\right)\right\} \leqslant A\left(\|h\|_{\alpha \beta \gamma}\right) .
$$

Proof. We can derive this lemma from estimate (4.2) in the same manner as Proposition 3.2.5 in Gyöngy and Pardoux (1993) is proved. For convenience' sake we reproduce the argument below. Clearly

$$
\begin{aligned}
\mathrm{E}\left\{\exp \left(\int_{0}^{T} \int_{0}^{1} h(t, x, u(t, x)) \mathrm{d} x \mathrm{~d} t\right)\right\} & =\sum_{n=0}^{\infty} \frac{1}{n !} \mathrm{E}\left\{\left(\int_{0}^{T} \int_{0}^{1} h(t, x, u(t, x)) \mathrm{d} x \mathrm{~d} t\right)^{n}\right\} \\
& =1+\sum_{n=1}^{\infty} I_{n},
\end{aligned}
$$

where

$$
\begin{aligned}
I_{n}:= & \mathrm{E}\left(\frac{1}{n !} \prod_{i=1}^{n} \int_{0}^{T} \int_{0}^{1} h\left(t_{i}, x, u\left(t_{i}, x\right)\right) \mathrm{d} x \mathrm{~d} t_{i}\right) \\
= & \mathrm{E}\left(\int_{0}^{T} \int_{t_{1}}^{T} \cdots \int_{t_{n-1}}^{T} \int_{0}^{1} h\left(t_{1}, x, u\left(t_{1}, x\right)\right) \mathrm{d} x \cdots \int_{0}^{1} h\left(t_{n}, x, u\left(t_{n}, x\right)\right) \mathrm{d} x \mathrm{~d} t_{n} \cdots \mathrm{d} t_{1}\right) \\
= & \int_{0}^{T} \int_{t_{1}}^{T} \cdots \int_{t_{n-2}}^{T} \mathrm{E}\left\{\int_{0}^{1} h\left(t_{1}, x, u\left(t_{1}, x\right)\right) \mathrm{d} x \cdots \int_{0}^{1} h\left(t_{n-1}, x, u\left(t_{n-1}, x\right)\right) \mathrm{d} x\right. \\
& \left.\times \mathrm{E}\left(\int_{t_{n}-1}^{T} \int_{0}^{1} h\left(t_{n}, x, u\left(t_{n}, x\right)\right) \mathrm{d} x \mathrm{~d} t_{n} \mid \mathscr{F}_{t_{n-1}}\right) \mathrm{d} t_{n-1} \cdots \mathrm{d} t_{1}\right\} .
\end{aligned}
$$

By Lemma 4.1,

$$
\begin{aligned}
I_{n} \leqslant & K \int_{0}^{T} \int_{t_{1}}^{T} \cdots \int_{t_{n-2}}^{T} \mathrm{E}\left\{\int_{0}^{1} h\left(t_{1}, x, u\left(t_{1}, x\right)\right) \mathrm{d} x \cdots \int_{0}^{1} h\left(t_{n-1}, x, u\left(t_{n-1}, x\right)\right) \mathrm{d} x\right. \\
& \left.\times\left(\int_{t_{n-1}}^{T}\left\|h\left(t_{n}\right)\right\|_{\alpha \beta}^{\gamma} \mathrm{d} t_{n}\right)^{1 / \gamma}\right\} \mathrm{d} t_{n-1} \cdots \mathrm{d} t_{1} \\
= & K \int_{0}^{T} \int_{t_{1}}^{T} \cdots \int_{t_{n-3}}^{T} \mathrm{E}\left\{\int_{t_{n-2}}^{T} \int_{0}^{1} h\left(t_{1}, x, u\left(t_{1}, x\right)\right) \mathrm{d} x \cdots \int_{0}^{1} h\left(t_{n-1}, x, u\left(t_{n-1}, x\right)\right) \mathrm{d} x\right. \\
& \left.\times\left(\int_{t_{n-1}}^{T}\left\|h\left(t_{n}\right)\right\|_{\alpha \beta}^{\gamma} \mathrm{d} t_{n}\right)^{1 / \gamma} \mathrm{d} t_{n-1}\right\} \mathrm{d} t_{n-2} \cdots \mathrm{d} t_{1} .
\end{aligned}
$$


Proceeding as before, we take conditional expectations successively with respect to $\mathscr{T}_{t_{n-2}}$, $\mathscr{F}_{t_{n-3}}, \ldots, \mathscr{F}_{t_{1}}$ and we obtain

$$
\begin{aligned}
I_{n} \leqslant & K^{n-1} \mathrm{E}\left\{\int_{0}^{T} \int_{0}^{1} h\left(t_{1}, x, u\left(t_{1}, x\right)\right) \mathrm{d} x\right. \\
& \left.\times\left(\int_{t_{1}}^{T} \cdots \int_{t_{n-1}}^{T}\left\|h\left(t_{2}\right)\right\|_{\alpha \beta}^{\gamma} \cdots\left\|h\left(t_{n}\right)\right\|_{\alpha \beta}^{\gamma} \mathrm{d} t_{n} \cdots \mathrm{d} t_{2}\right)^{1 / \gamma} \mathrm{d} t_{1}\right\} .
\end{aligned}
$$

Conditioning, finally, by $\mathscr{F}_{0}$, we get

$$
\begin{aligned}
I_{n} & \leqslant K^{n} \mathrm{E}\left\{\left(\int_{0}^{T} \int_{t_{1}}^{T} \cdots \int_{t_{n-1}}^{T}\left\|h\left(t_{1}\right)\right\|_{\alpha \beta}^{\gamma} \cdots\left\|h\left(t_{n}\right)\right\|_{\alpha \beta}^{\gamma} \mathrm{d} t_{n} \cdots \mathrm{d} t_{1}\right)^{1 / \gamma}\right\} \\
& =K^{n}\left(\frac{1}{n !}\right)^{1 / \gamma}\|h\|_{\alpha \beta \gamma}^{n} .
\end{aligned}
$$

Consequently, we can take

$$
A(y):=\sum_{n=0}^{\infty} K^{n}\left(\frac{1}{n !}\right)^{1 / \gamma} y^{n},
$$

which is well defined for every $y \in \mathbb{R}$.

Proposition 4.3. Let $f:=f(t, x, z)$ be a bounded Borel function such that

$$
|f(t, x, z)|^{2} \leqslant C+F(t, x, z)
$$

with some constant $C$ and Borel function $F \in L^{p q r}$ for some $p>1, q>2, r>4 q /(3 q-2)$. Let $\xi=\xi(t, x)$ be an $\mathscr{F}_{t}$-adapted random field, such that $\int_{0}^{T} \int_{0}^{1}|\xi(t, x)|^{2} \mathrm{~d} x \mathrm{~d} t \leqslant M$ (a.s.) for some constant $M$. Assume conditions $(B)$ and $(C)$. Then the solution $u$ of $\operatorname{Eq}\left(u_{0}, f+\xi, \sigma\right)$ satisfies the following estimates.

(i) Let $g=g(t, \omega, x, z)$ be an $\mathscr{F}_{t}$-adapted random field such that

$$
|g(t, \omega, x, z)|^{2} \leqslant \eta(t, \omega, x)+G(t, x, z),
$$

where $\eta=\eta(t, x)$ is some $\mathscr{F}_{t}$-adapted random field, $\int_{0}^{T} \int_{0}^{1} \eta \mathrm{d} x \mathrm{~d} t \leqslant L$ for some constant $L$, and $G \in L^{p^{\prime} q^{\prime} r^{\prime}}$, with $p^{\prime}>1, q^{\prime}>2, r^{\prime}>4 q^{\prime} /\left(3 q^{\prime}-2\right)$. Then

$$
Z(g):=\exp \left(-\int_{0}^{T} \int_{0}^{1} g(t, x, u(t, x)) W(\mathrm{~d} t, \mathrm{~d} x)-\frac{1}{2} \int_{0}^{T} \int_{0}^{1}|g(t, x, u(t, x))|^{2} \mathrm{~d} x \mathrm{~d} t\right)
$$

is a well-defined real random variable, and the measure $\tilde{P}$ given by $\mathrm{d} \tilde{P}=Z(g) \mathrm{d} P$ is a probability measure equivalent to $P$. Moreover, for every $\rho \in \mathbb{R}$, there exists a constant $K=K\left(T, C, L, M, p, q, r, p^{\prime}, q^{\prime}, r^{\prime},\|F\|_{p q r},\|G\|_{p^{\prime} q^{\prime} r^{\prime}}, \sigma, \rho\right)$ such that 


$$
\tilde{\mathrm{E}}\left(Z^{\rho}(g)\right) \leqslant K \quad \text { and } \quad \mathrm{E}\left(Z^{\rho}(g)\right) \leqslant K
$$

where $\tilde{\mathrm{E}}$ denotes expectation with respect to $\tilde{P}$.

(ii) For any $\alpha>1, \beta>2, \gamma>4 \beta /(3 \beta-2)$, there exists a constant $K=K(T, C, M$, $\left.p, q, r,\|F\|_{p q r}, \sigma, \alpha, \beta, \gamma\right)$ such that for every Borel function $h:[0, T] \times$ $[0,1] \times \mathbb{R} \rightarrow \mathbb{R}$,

$$
\mathrm{E}\left(\int_{0}^{T} \int_{0}^{1} h(t, x, u(t, x)) \mathrm{d} x \mathrm{~d} t\right) \leqslant K\|h\|_{\alpha \beta \gamma} .
$$

(iii) For any $\alpha>1, \beta>2$ and $\gamma>4 \beta /(3 \beta-2)$, there exists an analytic function $A: \mathbb{R}_{+} \rightarrow(0, \infty)$, depending on $T, C, M, p, q, r,\|F\|_{p q r}, \sigma, \alpha, \beta, \gamma$, such that, for every Borel function $h=h(t, x, z), h \in L^{\alpha \beta \gamma}$,

$$
\mathrm{E}\left\{\exp \left(\int_{0}^{T} \int_{0}^{1} h(t, x, u(t, x)) \mathrm{d} x \mathrm{~d} t\right)\right\} \leqslant A\left(\|h\|_{\alpha \beta \gamma}\right) .
$$

(iv) For every $\rho>1$ and $\delta>0$, there exists a constant $K=K(T, C, M, p, q, r$, $\left.\|F\|_{p q r}, \sigma, \rho, \delta\right)$ such that

$$
\mathrm{E}\left\{\sup _{(t, x) \in[0, T] \times[0,1]} \exp \left(-\delta\left\|u_{0}\right\|_{\infty}\right)|u(t, x)|^{\rho}\right\} \leqslant K,
$$

where $\left\|u_{0}\right\|_{\infty}:=\sup _{x \in[0,1]}\left|u_{0}(x)\right|$.

Proof. (i) Assume first that $g$ is bounded. Then $Z(g)$ is well defined, $\tilde{P}$ is a probability measure, and under $\bar{P}$, defined by $\mathrm{d} \bar{P}=Z(\bar{f}) \mathrm{d} P, \bar{f}:=\sigma^{-1}(f+\xi)$, the random field $u$ solves $\mathrm{Eq}\left(u_{0}, 0, \sigma\right)$ with the Brownian sheet

$$
\bar{W}(t, x):=W(t, x)+\int_{0}^{t} \int_{0}^{x} \bar{f}(s, y, u(s, y)) \mathrm{d} y \mathrm{~d} s
$$

in place of $W$. Moreover,

$$
\begin{aligned}
\overline{\mathrm{E}}\left[Z^{\rho}(g)\right] & =\overline{\mathrm{E}}\left\{\exp \left(-\rho \int_{0}^{T} \int_{0}^{1} g(t, x, u(t, x)) \mathrm{d} \bar{W}(t, x)-\rho^{2} \int_{0}^{T} \int_{0}^{1}|g(t, x, u(t, x))|^{2} \mathrm{~d} x \mathrm{~d} t\right) I\right\} \\
& \leqslant\{\overline{\mathrm{E}} \bar{Z}(2 \rho g)\}^{1 / 2}\left\{\overline{\mathrm{E}} I^{2}\right\}^{1 / 2}=\left\{\overline{\mathrm{E}} I^{2}\right\}^{1 / 2}
\end{aligned}
$$

where $\bar{Z}(2 \rho g)$ is defined by (4.17) with $\bar{W}$ and $2 \rho g$ in place of $W$ and $g$, respectively, $\overline{\mathrm{E}}$ denotes the expectation with respect to $\bar{P}$, and

$$
\begin{aligned}
I & :=\exp \left\{\left(\rho^{2}-\frac{\rho}{2}\right) \int_{0}^{T} \int_{0}^{1}|g(t, x, u(t, x))|^{2} \mathrm{~d} x \mathrm{~d} t+\rho \int_{0}^{T} \int_{0}^{1} g \bar{f}(t, x, u(t, x)) \mathrm{d} x \mathrm{~d} t\right\} \\
& \leqslant N \exp \left\{\left(\rho^{2}+|\rho|\right) \int_{0}^{T} \int_{0}^{1}\left(G(t, x, u(t, x))+\lambda^{2} F(t, x, u(t, x))\right) \mathrm{d} x \mathrm{~d} t\right\},
\end{aligned}
$$


with $\quad N:=\exp \left\{\left(\rho^{2}+|\rho|\right)\left(L+\lambda^{2}(C T+M)\right)\right\}, \quad \lambda:=\sup \left\{\left|\sigma^{-1}(t, x, z)\right|:(t, x, z) \in[0, T] \times\right.$ $[0,1] \times \mathbb{R}\}$. Hence, by Lemma 4.2 ,

$$
\overline{\mathrm{E}}\left(Z^{\rho}(g)\right) \leqslant N A^{1 / 4}\left(4\left(\rho^{2}+|\rho|\right)\|G\|_{p^{\prime} q^{\prime} r^{\prime}}\right) A^{1 / 4}\left(4\left(\rho^{2}+|\rho|\right) \lambda^{2}\|F\|_{p q r}\right),
$$

and by

$$
\mathrm{E}\left(Z^{\rho}(g)\right) \leqslant\left\{\overline{\mathrm{E}}\left(Z^{-2}(\bar{f})\right)\right\}^{1 / 2}\left\{\overline{\mathrm{E}}\left(Z^{2 \rho}(g)\right)\right\}^{1 / 2}
$$

we obtain (i) in the case of bounded $g$. We can treat the general case of unbounded $g$ after proving statement (ii).

(ii) Assume $h$ is non-negative. If $\rho, \delta>1,1 / \rho+1 / \delta=1$,

$$
\begin{aligned}
\mathrm{E}\left(\int_{0}^{T} \int_{0}^{1} h(t, x, u(t, x)) \mathrm{d} x \mathrm{~d} t\right) & =\overline{\mathrm{E}}\left(Z^{-1}(\bar{f}) \int_{0}^{T} \int_{0}^{1} h(t, x, u(t, x)) \mathrm{d} x \mathrm{~d} t\right) \\
& \leqslant T^{\delta-1}\left\{\overline{\mathrm{E}}\left(Z^{-\rho}(\bar{f})\right)\right\}^{1 / \rho}\left\{\overline{\mathrm{E}}\left(\int_{0}^{T} \int_{0}^{1} h(t, x, u(t, x))^{\delta} \mathrm{d} x \mathrm{~d} t\right)\right\}^{1 / \delta} \\
& \leqslant K\|h\|_{\delta \alpha, \delta \beta, \delta \gamma},
\end{aligned}
$$

where $K=K\left(T, C, M, p, q, r,\|F\|_{p q r}, \sigma, \delta, \alpha, \beta, \gamma\right)$, by estimate (i) and Lemma 4.2, for any $\alpha>1, \beta>2$ and $\gamma>4 \beta /(3 \beta-2)$. Since $\delta$ can be taken arbitrarily close to 1 , inequality (4.19) follows. We can now prove (i) without the additional condition that $g$ is bounded. We have (i) for $g_{n}:=g \mathbf{1}_{\{|g| \leqslant n\}}$ in place of $g$ with constant $K$ independent of $n$. Hence $Z^{\rho}\left(g_{n}\right)$ is uniformly integrable for every $\rho$. Using (ii), we can see that $Z(g)$ is well defined and that $Z\left(g_{n}\right) \rightarrow Z(g)$ as $n \rightarrow \infty$. Hence we obtain (i) by Fatou's lemma. Moreover, we obtain $\mathrm{E}(Z(g))=1$.

(iii) We assume $h$ is non-negative. By (i),

$$
\begin{aligned}
\mathrm{E}\left\{\exp \left(\int_{0}^{T} \int_{0}^{1} h(t, x, u(t, x)) \mathrm{d} x \mathrm{~d} t\right)\right\} & =\overline{\mathrm{E}}\left\{Z^{-1}(\bar{f}) \exp \left(\int_{0}^{T} \int_{0}^{1} h(t, x, u(t, x)) \mathrm{d} x \mathrm{~d} t\right)\right\} \\
& \leqslant\left\{\overline{\mathrm{E}} Z^{-2}(\bar{f})\right\}^{1 / 2}\left\{\overline{\mathrm{E}} \exp \left(2 \int_{0}^{T} \int_{0}^{1} h(t, x, u(t, x)) \mathrm{d} x \mathrm{~d} t\right)\right\}^{1 / 2} \\
& \leqslant K\left\{\overline{\mathrm{E}} \exp \left(2 \int_{0}^{T} \int_{0}^{1} h(t, x, u(t, x)) \mathrm{d} x \mathrm{~d} t\right)\right\}^{1 / 2}
\end{aligned}
$$

where $K=K\left(T, C, M, p, q, r,\|F\|_{p q r}, \sigma\right)$. From Lemma 4.2,

$$
\overline{\mathrm{E}}\left\{\exp \left(2 \int_{0}^{T} \int_{0}^{1} h(t, x, u(t, x)) \mathrm{d} x \mathrm{~d} t\right)\right\} \leqslant A\left(\|h\|_{\alpha \beta \gamma}\right),
$$

for some analytic function $A=A(T, \sigma, \alpha, \beta, \gamma): \mathbb{R}_{+} \rightarrow(0, \infty)$. 
(iv) Note that $u(t, x)=\eta(t, x)+\zeta(t, x)$, where

$\eta(t, x):=\int_{0}^{1} G_{t}(x, y) u_{0}(y) \mathrm{d} y, \quad \zeta(t, x):=\int_{0}^{t} \int_{0}^{1} G_{t-s}(x, y) \sigma(s, y, u(s, y)) \mathrm{d} \bar{W}(s, y)$,

$G$ is the heat kernel, and $\bar{W}(t, x)$ is defined by (4.22). We have

$$
|\eta(t, x)| \leqslant\left\|u_{0}\right\|_{\infty} \int_{0}^{1} G_{t}(x, y) \mathrm{d} y \leqslant\left\|u_{0}\right\|_{\infty}
$$

and

$$
\overline{\mathrm{E}}\left(\sup _{(t, x) \in[0, T] \times[0,1]}|\zeta(t, x)|^{m}\right) \leqslant K_{m}, \quad \forall m>0,
$$

by the Burkholder-Davis-Gundy inequality and Kolmogorov's theorem, where $K_{m}$ is a constant depending only on $m, T$ and $\sigma$. Applying estimate (i),

$$
\begin{aligned}
& \mathrm{E}\left\{\exp \left(-\delta\left\|u_{0}\right\|_{\infty}\right) \sup _{(t, x) \in[0, T] \times[0,1]}|u(t, x)|^{\rho}\right\} \\
& \quad=\overline{\mathrm{E}}\left\{Z^{-1}(\bar{f}) \exp \left(-\delta\left\|u_{0}\right\|_{\infty}\right) \sup _{(t, x) \in[0, T] \times[0,1]}|u(t, x)|^{\rho}\right\} \\
& \quad \leqslant\left\{\overline{\mathrm{E}} Z^{-2}(\bar{f})\right\}^{1 / 2}\left\{\overline{\mathrm{E}} \exp \left(-2 \delta\left\|u_{0}\right\|_{\infty}\right) \sup _{(t, x) \in[0, T] \times[0,1]}|u(t, x)|^{2 \rho}\right\}^{1 / 2} \leqslant K,
\end{aligned}
$$

for some constant $K=K\left(T, C, M, p, q, r,\|F\|_{p q r}, \sigma, \rho, \delta\right)$.

Remark 4.4. Let $u_{n}=u_{n}(t, x)$ be a sequence of $\mathscr{F}_{t}$-adapted random fields on $[0,1]$, such that $u_{n}(t, x) \rightarrow v(t, x)$ (a.s.) for every $t \in[0, T], x \in[0,1]$ for some random field $v$. Assume that estimates (i), (ii), (iii) of Proposition 4.3 hold with $u_{n}$ in place of $u$, with the same constant $K$ and function $A$, independently of $n$. Then estimates (i), (ii), (iii) hold with $v$ in place of $u$. Assume that $u_{n}(0, x)$ is continuous in $x$ and converges uniformly in $x$. Assume, moreover, that for every $n$ there exists a random variable $\eta_{n}$ and that, for some $\delta>0, \rho>0$, there exists a constant $K$ such that

$$
P\left(\left|u_{n}(t, x)\right| \leqslant \eta_{n} \forall(t, x) \in[0, T] \times[0,1]\right)=1 \quad \text { and } \mathrm{E}\left(\exp \left(-\delta\left\|u_{n}(0)\right\|_{\infty}\right)\left|\eta_{n}\right|^{\rho}\right) \leqslant K,
$$

for all $n$. Then

$$
P\left(|v(t, x)| \leqslant \liminf _{n} \eta_{n}:=\eta<\infty \forall t, x\right)=1, \quad \mathrm{E}\left(\exp \left(-\delta\|v(0)\|_{\infty}\right)|\eta|^{\rho}\right) \leqslant K .
$$

Proof. Estimates (i), (ii), (iii) for $v$ follow by Fatou's lemma from those for $u_{n}$ when $g$ and $h$ are bounded and continuous in $z \in \mathbb{R}$. Hence the general case can be obtained by the monotone class theorem. Estimate (4.35) follows from (4.34) by Fatou's lemma.

The following proposition generalizes a method used by Krylov for the construction of 
solutions to non-degenerate stochastic differential equations with measurable coefficients (see Krylov 1969; 1980). Though it is essentially the same as Proposition 3.3.2 and Corollary 3.3.3 in Gyöngy and Pardoux (1993), we present the proof, since the proof of Proposition 3.3.2 in Gyöngy and Pardoux (1993) contains a gap.

Proposition 4.5. Let $h_{n}=h_{n}(t, x, z)$ be a sequence of Borel functions such that

$$
\begin{gathered}
h_{n}(t, x, z) \rightarrow h(t, x, z) \quad d t \otimes d x \otimes d z \text { a.e., } \\
\left|h_{n}(t, x, z)\right| \leqslant C+M(t, x, z) \quad d t \otimes d x \otimes d z \text { a.e., } \quad \forall n \geqslant 1,
\end{gathered}
$$

for some Borel function $h=h(t, x, z)$, constant $C \geqslant 0$ and non-negative function $M$ belonging to $L^{\alpha^{\prime} \beta^{\prime} \gamma^{\prime}}([0, T] \times[0,1] \times \mathbb{R})$ for some $\alpha^{\prime}>1, \beta^{\prime}>2, \gamma^{\prime}>4 \beta^{\prime} /\left(3 \beta^{\prime}-2\right)$. Let $u_{n}=u_{n}(t, x)$ be a sequence of random fields on $[0, T] \times[0,1]$, such that

$$
u_{n}(t, x) \rightarrow u(t, x)(\text { a.s. }) \quad \forall t \in[0, T], \quad \forall x \in[0,1]
$$

and, for every $\alpha>1, \beta>2, \gamma>4 \beta /(3 \beta-2)$,

$$
\mathrm{E}\left(\int_{0}^{T} \int_{0}^{1}\left|g\left(t, x, u_{n}(t, x)\right)\right| \mathrm{d} x \mathrm{~d} t\right) \leqslant K\|g\|_{\alpha \beta \gamma}
$$

for all Borel functions $g=g(t, x, z)$, with a constant $K$ independent of $n$ and $g$. Then

$$
\lim _{n \rightarrow \infty} \mathrm{E}\left(\int_{0}^{T} \int_{0}^{1}\left|h_{n}\left(t, x, u_{n}(t, x)\right)-h(t, x, u(t, x))\right| \mathrm{d} x \mathrm{~d} t\right)=0 .
$$

Proof. First, note that by Remark 4.4 estimate (4.39) holds with $u$ in place of $u_{n}$. Let $\kappa: \mathbb{R}^{+} \rightarrow \mathbb{R}^{+}$be a smooth decreasing function, with $\kappa(0)=1$, and $\kappa(z)=0$ for $z \geqslant 1$. For every $\varepsilon>0$ and $R>0$, we can find a bounded continuous function $H_{\varepsilon R}$ such that

$$
\left\|\left(h-H_{\varepsilon R}\right) \mathbf{1}_{[-R, R]}(z)\right\|_{\alpha^{\prime} \beta^{\prime} \gamma^{\prime}}<\varepsilon .
$$

Clearly

$$
I(n):=\mathrm{E}\left(\int_{0}^{T} \int_{0}^{1}\left|h_{n}\left(u_{n}\right)-h(u)\right| \mathrm{d} x \mathrm{~d} s\right) \leqslant \sum_{j=1}^{5} I_{j},
$$

where 


$$
\begin{aligned}
& I_{1}:=\mathrm{E}\left(\int_{0}^{T} \int_{0}^{1} \kappa\left(\left|u_{n}\right| / R\right)\left|h_{n}\left(u_{n}\right)-h\left(u_{n}\right)\right| \mathrm{d} x \mathrm{~d} s\right), \\
& I_{2}:=\mathrm{E}\left(\int_{0}^{T} \int_{0}^{1}\left|H_{\varepsilon R}\left(u_{n}\right)-H_{\varepsilon R}(u)\right| \mathrm{d} x \mathrm{~d} s\right), \\
& I_{3}:=\mathrm{E}\left(\int_{0}^{T} \int_{0}^{1} \kappa\left(\left|u_{n}\right| / R\right)\left|h\left(u_{n}\right)-H_{\varepsilon R}\left(u_{n}\right)\right| \mathrm{d} x \mathrm{~d} s\right), \\
& I_{4}:=\mathrm{E}\left(\int_{0}^{T} \int_{0}^{1} \kappa(|u| / R)\left|h(u)-H_{\varepsilon R}(u)\right| \mathrm{d} x \mathrm{~d} s\right), \\
& I_{5}:=\mathrm{E}\left(\int_{0}^{T} \int_{0}^{1}\left\{1-\kappa\left(\left(\left|u_{n}\right| \vee|u|\right) / R\right)\right\}\left\{\left|h_{n}\left(u_{n}\right)\right|+|h(u)|\right\} \mathrm{d} x \mathrm{~d} s\right) .
\end{aligned}
$$

(We omit the integration variables for simplicity of notation.) By (4.39), we have

$$
\begin{aligned}
& I_{1} \leqslant K\left\|\left(h_{n}-h\right) \mathbf{1}_{[-R, R]}(z)\right\|_{\alpha^{\prime} \beta^{\prime} \gamma^{\prime}}, I_{3} \leqslant K\left\|\left(h-H_{\varepsilon R}\right) \mathbf{1}_{[-R, R]}(z)\right\|_{\alpha^{\prime} \beta^{\prime} \gamma^{\prime}}<\varepsilon, \\
& I_{4} \leqslant K\left\|\left(h-H_{\varepsilon R}\right) \mathbf{1}_{[-R, R]}(z)\right\|_{\alpha^{\prime} \beta^{\prime} \gamma^{\prime}}<\varepsilon .
\end{aligned}
$$

By Hölder's inequality we obtain

$$
I_{5} \leqslant L\left\{\mathrm{E}\left(\int_{0}^{T} \int_{0}^{1}\left(1-\kappa\left(\left(\left|u_{n}\right| \vee|u|\right) / R\right)\right)^{\rho} \mathrm{d} x \mathrm{~d} s\right)\right\}^{1 / \rho}
$$

with

$$
L:=\sup _{n}\left\{\left(\mathrm{E} \int_{0}^{T} \int_{0}^{1}\left(C+M\left(u_{n}\right)\right)^{\delta} \mathrm{d} x \mathrm{~d} s\right)^{1 / \delta}+\left(\mathrm{E} \int_{0}^{T} \int_{0}^{1}(C+M(u))^{\delta} \mathrm{d} x \mathrm{~d} s\right)^{1 / \delta}\right\}<\infty,
$$

by applying (4.39) with $\alpha:=\alpha^{\prime} / \delta, \beta:=\beta^{\prime} / \delta, \gamma:=\gamma^{\prime} / \delta, \delta>1$ small enough, $1 / \delta+$ $1 / \rho=1$. Hence, letting $n \rightarrow \infty$ in (4.42), we obtain

$$
\limsup _{n \rightarrow \infty} I(n) \leqslant L E\left(\int_{0}^{T} \int_{0}^{1}(1-\kappa(|u| / R))^{\rho} \mathrm{d} x \mathrm{~d} s\right)^{1 / \rho}+2 \varepsilon
$$

Now letting $R \rightarrow \infty$, we obtain $\lim \sup _{n \rightarrow \infty} I(n) \leqslant 2 \varepsilon$, which proves the proposition since $\varepsilon$ is arbitrarily small.

Corollary 4.6. Let $\sigma=\sigma(t, x, z)$ be a Borel function satisfying condition $(B)$. Let $f_{n}(t, x, z)$ be a sequence of Borel functions such that 


$$
\begin{gathered}
f_{n}^{2}(t, x, z) \leqslant C+F(t, x, z) \quad d t \otimes d x \otimes d z \text { a.e. } \forall n, \\
f_{n} \rightarrow f \quad d t \otimes d x \otimes d z \text { a.e. }
\end{gathered}
$$

where $C \geqslant 0$ is a constant, $F$ is a Borel function from $L^{p q r}$ for some $p>1, q>2$, $r>4 q /(3 q-2)$, and $f$ is some Borel function. Let $\xi_{n}:=\xi_{n}(t, x)$ be a sequence of $\mathscr{F}_{t^{-}}$ adapted random fields, such that

$$
\int_{0}^{T} \int_{0}^{1}\left|\xi_{n}(t, x)-\xi(t, x)\right| \mathrm{d} x \mathrm{~d} t \rightarrow 0 \text { (a.s.), }
$$

where $\xi$ is a random field such that $\xi \in L^{2}([0, T] \times[0,1])$ (a.s.). Let $u_{0 n}$ be a sequence of $\mathscr{F}_{0}$-measurable random variables in $C([0,1])$ converging almost surely to a random variable $u_{0}$ in $C([0,1])$. Assume that $\mathrm{Eq}\left(u_{0 n}, f_{n}+\xi_{n}, \sigma\right)$ admits a solution $u_{n}$ on $[0, T]$ such that, for every $\alpha>1, \beta>2, \gamma>4 \beta /(3 \beta-2)$,

$$
\mathrm{E}\left(\int_{0}^{T} \int_{0}^{1}\left|h\left(t, x, u_{n}(t, x)\right)\right| \mathrm{d} t \mathrm{~d} t\right) \leqslant K\|h\|_{\alpha \beta \gamma}
$$

for all Borel functions $h=h(t, x, z)$, with a constant $K$ independent of $n$ and $h$. Assume, moreover, that

$$
\begin{gathered}
\mathrm{E}\left(\sup _{(t, x) \in[0, T] \times[0,1]} \exp \left(-\delta\left\|u_{0 n}\right\|_{\infty}\right)\left|u_{n}(t, x)\right|^{\rho}\right) \leqslant C \quad \forall n, \\
u_{n}(t, x) \rightarrow u(t, x)(\text { a.s. }) \quad \forall t \in[0, T], \quad x \in[0,1],
\end{gathered}
$$

with some constants $\rho>1, \delta>0, C>0$ and random field $u$. Then $u$ has a continuous modification which is a solution of $\mathrm{Eq}\left(u_{0}, f+\xi, \sigma\right)$.

Proof. Fix $\varphi \in C^{2}([0,1])$ such that $\varphi(0)=\varphi(1)=0$. We have

$$
\begin{aligned}
\int_{0}^{1} u_{n}(t, x) \varphi(x) \mathrm{d} x= & \int_{0}^{1} u_{0 n}(x) \varphi(x) \mathrm{d} x+\int_{0}^{t} \int_{0}^{1} u_{n}(s, x) \varphi^{\prime \prime}(x) \mathrm{d} x+\int_{0}^{t} \int_{0}^{1} f_{n}\left(s, x, u_{n}(s, x)\right) \varphi(x) \mathrm{d} x \mathrm{~d} s \\
& +\int_{0}^{t} \int_{0}^{1} \xi_{n}(s, x) \varphi(x) \mathrm{d} x \mathrm{~d} s+\int_{0}^{t} \int_{0}^{1} \sigma\left(s, x, u_{n}(s, x)\right) \varphi(x) \mathrm{d} W(s, x) .
\end{aligned}
$$

Since $u_{0 n}$ converges uniformly to $u_{0}$ in $C([0,1])$ (a.s.)

$$
\lim _{n \rightarrow \infty} \int_{0}^{1} u_{0 n}(x) \varphi(x) \mathrm{d} x=\int_{0}^{1} u_{0}(x) \varphi(x) \mathrm{d} x, \text { a.s. }
$$

By assumptions (4.50) and (4.51),

$$
\begin{gathered}
\int_{0}^{1} u_{n}(t, x) \varphi(x) \mathrm{d} x \rightarrow \int_{0}^{1} u(t, x) \varphi(x) \mathrm{d} x, \\
\int_{0}^{t} \int_{0}^{1} u_{n}(s, x) \varphi^{\prime \prime}(x) \mathrm{d} x \rightarrow \int_{0}^{t} \int_{0}^{1} u(s, x) \varphi^{\prime \prime}(x) \mathrm{d} x
\end{gathered}
$$


in probability, for every $t \in[0, T]$. Clearly,

$$
\int_{0}^{t} \int_{0}^{1} \sigma\left(s, x, u_{n}(s, x)\right) \varphi(x) \mathrm{d} W(s, x) \rightarrow \int_{0}^{t} \int_{0}^{1} \sigma(s, x, u(s, x)) \varphi(x) \mathrm{d} W(s, x)
$$

in probability, and

$$
\int_{0}^{t} \int_{0}^{1} \xi_{n}(s, x) \varphi(x) \mathrm{d} x \mathrm{~d} s \rightarrow \int_{0}^{t} \int_{0}^{1} \xi_{n}(s, x) \varphi(x) \mathrm{d} x \mathrm{~d} s \text { (a.s.). }
$$

By the previous proposition

$$
\lim _{n \rightarrow \infty} \int_{0}^{t} \int_{0}^{1}\left|f_{n}\left(s, x, u_{n}(s, x)\right) \varphi(x)-f(s, x, u(s, x)) \varphi(x)\right| \mathrm{d} x \mathrm{~d} s \rightarrow 0
$$

in probability. Thus letting $n \rightarrow \infty$ in (4.52), we get (4.52) with $u$ in place of $u_{n}$ for each $t \in[0, T]$. Hence, by Proposition $3.1 u$ has a continuous modification, which is a solution of $\mathrm{Eq}\left(u_{0}, f+\xi, \sigma\right)$.

\section{Approximation, existence and uniqueness of the solutions}

The following approximation theorem is the key result of this paper.

Theorem 5.1. Assume $(A),(B)$ and $(C)$. Let $f_{n}:=f_{n}(t, x, z)$ be a sequence of bounded Borel functions, such that

$$
\begin{gathered}
f_{n}^{2}(t, x, z) \leqslant C+F(t, x, z) \quad(d t \otimes d x \otimes d z \text { a.e. }) \forall n, \\
f_{n} \rightarrow f \quad(d t \otimes d x \otimes d z \text { a.e. }),
\end{gathered}
$$

where $C \geqslant 0$ is a constant, $F$ is a Borel function from $L^{p q r}$ for some $p>1, q>2$, $r>4 q /(3 q-2)$, and $f$ is some Borel function. Let $\xi_{n}:=\xi_{n}(t, x)$ be a sequence of $\mathscr{F}_{t^{-}}$ adapted random fields on $[0, T] \times[0,1]$, such that

$$
\xi_{n} \rightarrow \xi(d t \otimes P \otimes d x \text { a.s. }), \quad\left|\xi_{n}(t, x)\right| \leqslant \xi(t, x)(d t \otimes P \otimes d x \text { a.s. }) \quad \forall n,
$$

where $\xi$, $\zeta$ are $\mathscr{F}_{t}$-adapted random fields, such that $\zeta \in L^{2}([0, T] \times[0,1])$ (a.s.). Let $u_{0 n}$ be a sequence of $\mathscr{F}_{0}$-measurable random variables in $C([0,1])$ converging almost surely to a random variable $u_{0}$ in $C([0,1])$. Then the solution $u_{n}$ of $\mathrm{Eq}\left(u_{0 n}, f_{n}+\xi_{n}, \sigma\right)$ on $[0, T]$ converges almost surely to a random field $u=u(t, x)$, uniformly in $(t, x) \in[0, T] \times[0,1]$, and $u$ is a solution of $\mathrm{Eq}\left(u_{0}, f+\xi, \sigma\right)$ which satisfies

$$
\int_{0}^{T} \int_{0}^{1} F(t, x, u(t, x)) \mathrm{d} x \mathrm{~d} t<\infty(\text { a.s. }) .
$$

Proof. We proceed in two steps.

Step 1. In addition to the conditions of the theorem assume that, for a constant $R$, 


$$
\int_{0}^{T} \int_{0}^{1}\left|\xi_{n}(t, x)\right|^{2} \mathrm{~d} x \mathrm{~d} t \leqslant R \text { (a.s.) } \quad \forall n .
$$

Define

$$
\begin{aligned}
\bar{f}_{n k}:=\bigwedge_{i=n}^{k} f_{i}, & \bar{f}_{(n)}:=\bigwedge_{i=n}^{\infty} f_{i}, & \bar{\xi}_{n k}:=\bigwedge_{i=n}^{k} \xi_{i}, & \bar{\xi}_{(n)}:=\bigwedge_{i=n}^{\infty} \xi_{i}, & \bar{u}_{0 n k}:=\bigwedge_{i=n}^{k} u_{0 i}, \\
f_{n k}:=\bigvee_{i=n}^{k} f_{i}, & f_{(n)}:=\bigvee_{i=n}^{\infty} f_{i}, & \xi_{n k}:=\bigvee_{i=n}^{k} \xi_{i}, & \xi_{(n)}:=\bigvee_{i=n}^{\infty} \xi_{i}, & u_{0 n k}:=\bigvee_{i=n}^{k} u_{0 i} .
\end{aligned}
$$

Notice that:

(a) $\bar{f}_{n k}$ and $f_{n k}$ are bounded;

(b) $\left\{\bar{f}_{n k}\right\}_{k},\left\{\bar{\xi}_{n k}\right\}_{k},\left\{\bar{u}_{0 n k}\right\}_{k}$ are decreasing and $\left\{f_{n k}\right\}_{k},\left\{\xi_{n k}\right\}_{k},\left\{u_{0 n k}\right\}_{k}$ are increasing;

(c) $f_{n k} \geqslant f_{m k} \geqslant \bar{f}_{m k} \geqslant \bar{f}_{n k}, \xi_{n k} \geqslant \xi_{m k} \geqslant \bar{\xi}_{m k} \geqslant \bar{\xi}_{n k}$ and $u_{0 n k} \geqslant u_{0 m k} \geqslant \bar{u}_{0 m k} \geqslant \bar{u}_{0 n k}$ for $n \leqslant m \leqslant k$.

Let $\bar{u}_{n k}$ and $u_{n k}$ be the unique solutions to $\operatorname{Eq}\left(\bar{u}_{0 n k}, \bar{f}_{n k}, \sigma\right)$ and $\mathrm{Eq}\left(u_{0 n k}, f_{n k}, \sigma\right)$, respectively. Using the comparison result of Proposition 3.3, $\left\{\bar{u}_{n k}\right\}_{k}$ is decreasing, $\left\{u_{n k}\right\}_{k}$ is increasing, and

$$
u_{n k} \geqslant u_{m k} \geqslant \bar{u}_{m k} \geqslant \bar{u}_{n k}, \quad \text { if } n \leqslant m \leqslant k .
$$

By Proposition 4.3 there exists a constant $K$, independent of $n$ and $k$, such that

$$
\begin{aligned}
& \mathrm{E}\left(\sup _{t, x} \exp \left(-\left\|\bar{u}_{0 n k}\right\|_{\infty}\right)\left|\bar{u}_{n k}(t, x)\right|^{2}\right) \leqslant K, \\
& \mathrm{E}\left(\sup _{t, x} \exp \left\{-\left\|u_{0 n k}\right\|_{\infty}\right\}\left|u_{n k}(t, x)\right|^{2}\right) \leqslant K .
\end{aligned}
$$

This implies that the limits

$$
\bar{u}_{(n)}(t, x):=\lim _{k \rightarrow \infty} u_{n k}(t, x), \quad u_{(n)}(t, x):=\lim _{k \rightarrow \infty} u_{n k}(t, x)
$$

are almost surely bounded random fields. Clearly, the limits

$$
\bar{u}_{(0 n)}(t, x):=\lim _{k \rightarrow \infty} u_{0 n k}(x), \quad u_{(0 n)}(x):=\lim _{k \rightarrow \infty} u_{0 n k}(x)
$$

are almost surely continuous random fields on $[0,1]$. By Proposition 4.3 and Corollary 4.6, $\bar{u}_{(n)}$ and $u_{(n)}$ have continuous modifications, denoted also by $\bar{u}_{(n)}$ and $u_{(n)}$, which are solutions of $\operatorname{Eq}\left(\bar{u}_{(0 n)}, \bar{f}_{(n)}+\bar{\xi}_{(n)}, \sigma\right)$ and $\operatorname{Eq}\left(u_{(0 n)}, f_{(n)}+\xi_{(n)}, \sigma\right)$, respectively. Letting $k \rightarrow \infty$ in (5.6), we find that

$$
u_{(n)} \geqslant u_{(m)} \geqslant \bar{u}_{(m)} \geqslant \bar{u}_{(n)}, \quad \text { if } n \leqslant m,
$$

which implies that the random fields $u:=\lim _{n \rightarrow \infty} u_{(n)}$ and $\bar{u}:=\lim _{n \rightarrow \infty} u_{(n)}$ are well defined, and almost surely bounded by virtue of Remark 4.4. Moreover, using Proposition 4.3, Remark 
4.4 and Corollary 4.6, we deduce that $\bar{u}$ and $u$ have continuous modifications, denoted also by $\bar{u}$ and $u$, which are solutions of $\operatorname{Eq}\left(u_{0}, f+\xi, \sigma\right)$. Clearly, $\bar{u} \leqslant u$ (a.s.), and by Girsanov's theorem $\bar{u}$ and $u$ must have the same law, which implies $\bar{u}=u$. For $n \leqslant i \leqslant k$, we have

$$
\bar{f}_{n k} \leqslant f_{i} \leqslant f_{n k}, \quad \bar{\xi}_{n k} \leqslant \xi_{i} \leqslant \xi_{n k}, \quad \bar{u}_{0 n k} \leqslant u_{0 i} \leqslant u_{0 n k} .
$$

Hence $\bar{u}_{n k} \leqslant u_{i} \leqslant u_{n k}$ by Proposition 3.3, which implies

$$
\bar{u}_{n k} \leqslant \bigwedge_{i=n}^{k} u_{i} \leqslant \bigvee_{i=n}^{k} u_{i} \leqslant u_{n k}
$$

Taking $k \rightarrow \infty$ and then $n \rightarrow \infty$, we obtain

$$
\bar{u} \leqslant \liminf _{n \rightarrow \infty} u_{n} \leqslant \limsup _{n \rightarrow \infty} u_{n} \leqslant u=\bar{u}
$$

Therefore, $u_{n} \rightarrow \bar{u}=u$ (a.s.) for all $t$ and $x$. The convergence is uniform in $(t, x) \in$ $[0, T] \times[0,1]$, since the convergences $\bar{u}_{n k} \rightarrow \bar{u}_{(n)}, \bar{u}_{(n)} \rightarrow \bar{u}, u_{n k} \rightarrow u_{(n)}$ and $u_{(n)} \rightarrow u$ are uniform by virtue of Dini's theorem. Moreover, using Proposition 4.3 and Remark 4.4, we obtain

$$
\mathrm{E}\left(\int_{0}^{T} \int_{0}^{1} F(t, x, u(t, x)) \mathrm{d} x \mathrm{~d} t\right)<\infty
$$

Step 2. We now consider the case when we do not have condition (5.4). Define the stopping time

$$
\tau_{R}:=\inf \left\{t \in[0, T]: \int_{0}^{t} \int_{0}^{1}|\zeta(s, x)|^{2} \mathrm{~d} x \mathrm{~d} s \geqslant R\right\}
$$

and the random field $\xi_{n}^{(R)}=\xi_{n}^{(R)}(t, x):=\xi_{n}\left(t \wedge \tau_{R}, x\right)$ for every $R>0$. Then, by step 1 , the solution $u_{n}^{(R)}$ of $\operatorname{Eq}\left(u_{0 n}, f_{n}+\xi_{n}^{(R)}, \sigma\right)$ converges almost surely in $C([0, T] \times[0,1])$ to a random field $u^{(R)}$, which is a solution of $\mathrm{Eq}\left(u_{0}, f+\xi^{(R)}, \sigma\right)$, where $\xi^{(R)}(t, x):=\xi\left(t \wedge \tau_{R}, x\right)$. Note that if $Q \leqslant R$ then $\tau_{Q} \leqslant \tau_{R}$, and almost surely $u_{n}^{(Q)}(t, x)=u_{n}^{(R)}(t, x)=u_{n}(t, x)$ for all $t \in\left[0, \tau_{Q} \wedge T\right], \quad x \in[0,1]$. Consequently, almost surely $u^{(Q)}(t, x)=u^{(r)}(t, x)$ for all $t \in\left[0, \tau_{Q} \wedge T\right], x \in[0,1]$. Hence taking into account that $\tau_{R} \uparrow \infty$ as $R \rightarrow \infty$, we can define $u(t, x):=\lim _{R \rightarrow \infty} u^{(R)}(t, x)$ for all $t \in[0, T], x \in[0,1]$. Moreover, we obtain that $u_{n}$ converges almost surely in $C([0, T] \times[0,1])$ to $u$, which is a solution on $[0, T]$ of $\mathrm{Eq}\left(u_{0}, f, \sigma\right)$. Finally, note that, for each $N>0$ and $R>0$, 


$$
\begin{aligned}
& P\left(\int_{0}^{T} \int_{0}^{1} F(t, x, u(t, x)) \mathrm{d} x \mathrm{~d} t \geqslant N\right) \\
& \quad \leqslant P\left(\int_{0}^{T} \int_{0}^{1} F\left(t, x, u^{(R)}(t, x)\right) \mathrm{d} x \mathrm{~d} t \geqslant N\right)+P\left(\tau_{R}<T\right) \\
& \quad \leqslant \frac{1}{N} \mathrm{E}\left(\int_{0}^{T} \int_{0}^{1} F\left(t, x, u^{(R)}(t, x)\right) \mathrm{d} x \mathrm{~d} t\right)+P\left(\tau_{R}<T\right) \leqslant \frac{K_{R}}{N}+P\left(\tau_{R}<T\right)
\end{aligned}
$$

with a constant $K_{R}$ by virtue of (5.14) in step 1 . Letting $N \rightarrow \infty$ and then $R \rightarrow \infty$, we obtain

$$
P\left(\int_{0}^{T} \int_{0}^{1} F(t, x, u(t, x)) \mathrm{d} x \mathrm{~d} t=\infty\right)=0,
$$

that is to say (5.3) holds.

The existence of a solution to $\operatorname{Eq}\left(u_{0}, f, \sigma\right)$ follows immediately from the above theorem under conditions (A), (B) and (C). In order to deal with the question of uniqueness the following definition is useful.

Definition 5.2. Assume conditions $(A),(B)$ and $(C)$. We say that a solution $u(t, x)$ of $\mathrm{Eq}\left(u_{0}, f, \sigma\right)$ on $[0, T]$ is constructible, if there exist a sequence of bounded Borel functions $f_{n}=f_{n}(t, x, z)$ and a sequence of random fields $\xi_{n}=\xi_{n}(t, x)$, satisfying the conditions of Theorem 5.1, and $u_{n}(t, x) \rightarrow u(t, x)$ (a.s.) for every $t \in[0, T], x \in[0,1]$, where $u_{n}$ is the solution of $\mathrm{Eq}\left(u_{0}, f_{n}+\xi_{n}, \sigma\right)$ on $[0, T]$.

Proof of Theorem 2.1. Applying Theorem 5.1 with $f_{n}:=f \mathbf{1}_{\{|f| \leqslant n\}}, \xi_{n}=0$, we obtain the existence of a constructible solution satisfying the required estimate. Let $u$ and $v$ be constructible solutions, that is, $u_{n} \rightarrow u, v_{n} \rightarrow v$, where $u_{n}$ and $v_{n}$ are the solutions of $\operatorname{Eq}\left(u_{0}, f_{n}+\xi_{n}, \sigma\right)$ and $\operatorname{Eq}\left(u_{0}, g_{n}+\eta_{n}, \sigma\right)$, respectively, for appropriate sequences $f_{n}, \xi_{n}, g_{n}$, $\eta_{n}$. Define the sequences $h_{n}:=f_{n}, \zeta_{n}:=\xi_{n}$ for $n$ even, and $h_{n}:=g_{n}, \zeta_{n}:=\eta_{n}$ for $n$ odd. Then, by Theorem 5.1, the solution $w_{n}$ to $\operatorname{Eq}\left(u_{0}, h_{n}+\zeta_{n}, \sigma\right)$ converges to some random field $w$, which implies $u=v$. Let $u$ be now a solution of $\operatorname{Eq}\left(u_{0}, f, \sigma\right)$ such that (5.3) holds. For $n \geqslant 1$, define

$$
f_{n}:=f \mathbf{1}_{\{|f| \leqslant n\}}, \quad \xi_{n}(t, x):=f(t, x, u(t, x))-f_{n}(t, x, u(t, x)) .
$$

Then clearly $f_{n}, \xi_{n}$ satisfy the condition of Theorem 5.1 and $u$ is a solution of $\mathrm{Eq}\left(u_{0}, f_{n}+\xi_{n}, \sigma\right)$ for every $n$. Consequently, $u$ is a constructible solution, which proves uniqueness. In order to show that $u$ is a $C([0,1])$-valued Markov process, let us consider a $C([0,1])$-valued $\mathscr{F}_{0}$-adapted sequence of random variables $u_{0 n}$ converging almost surely to $u_{0}$ in $C([0,1])$. Let $u_{n}$ denote the solution of $\operatorname{Eq}\left(u_{0 n}, f, \sigma\right)$. Clearly $u_{n}$ is the unique solution of $\operatorname{Eq}\left(u_{0 n}, f_{n}+\xi_{n}, \sigma\right)$, where $f_{n}:=f \mathbf{1}_{\{|f| \leqslant n\}}$ and $\xi_{n}(t, x):=f\left(t, x, u_{n}(t, x)\right)-f_{n}(t, x$, 
$\left.u_{n}(t, x)\right)$. Therefore, by Theorem 5.1 , we obtain that $u_{n}$ converges almost surely in $C([0, T] \times[0,1])$ to $u$. Hence the Markov property of $\{u(t): t \in[0, T]\}$ follows by standard arguments.

Proof of Theorem 2.3. Define $\bar{f}_{n}:=(\bar{f} \wedge n) \vee(-n), f_{n}:=(f \wedge n) \vee(-n)$. Clearly, $\bar{f}_{n} \leqslant f_{n}$ for every integer $n \geqslant 1$. Hence, by Proposition 3.3, we have $\bar{u}_{n}(t, x) \leqslant u_{n}(t, x)$ for the solutions $\bar{u}_{n}$ and $u_{n}$ of $\operatorname{Eq}\left(u_{0}, \bar{f}_{n}, \sigma\right)$ and $\operatorname{Eq}\left(u_{0}, f_{n}, \sigma\right)$, respectively. By Theorem $5.1 u_{n} \rightarrow u$ and $\bar{u}_{n} \rightarrow \bar{u}$. Consequently, almost surely $\bar{u}(t, x) \leqslant u(t, x)$ for all $t, x$.

\section{Acknowledgement}

Part of this work was done while the first named author was visiting the Department of Mathematics and Statistics of the University of Edinburgh, and was supported by grants PB96-0087, PB96-1182 of CICYT, 1997SGR00144 of CIRIT, and his visit was sponsored by SEUID.

\section{References}

Bally, V., Gyöngy, I. and Pardoux, E. (1994) White noise driven parabolic SPDEs with measurable drift. J. Funct. Anal., 120, 484-510.

Da Prato, G. and Zabczyk, J. (1992) Stochastic equations in infinite dimensions, Encyclopedia of Mathematics and Its Applications 44. Cambridge: Cambridge University Press.

Dellacherie C. and Meyer, P.-A. (1978) Probabilities and Potential. Amsterdam: North-Holland.

Gyöngy, I. (1998) Existence and uniqueness results for semilinear stochastic partial differential equations. Stochastic Process. Appl., 73, 271-299.

Gyöngy, I. and Pardoux, E. (1992) Weak and strong solutions of white noise driven SPDEs. Preprint No. 22/92, Laboratoire de Mathématiques Marseille, Université de Provence.

Gyöngy, I. and Pardoux, E. (1993) On the regularization effect of space-time white noise on quasilinear parabolic partial differential equations. Probab. Theory Related Fields, 97, 211-229.

Krylov, N.V. (1969) On Itô's stochastic integral equations. Theory Probab. Appl., 120, 330-336.

Krylov, N.V. (1980) Controlled Diffusion Processes. Berlin: Springer-Verlag.

Rozovskii, B.L. (1990) Stochastic Evolution Systems. Dordrecht: Kluwer.

Walsh, J.B. (1986) An introduction to stochastic partial differential equations. In P.L. Hennequin (ed.), École d'Été de Probabilités de Saint Flour XIV, Lecture Notes in Math. 1180, pp. 265-437. Berlin: Springer-Verlag.

Received September 1999 and revised March 2000. 